\title{
Human CT Measurements of Structure/Electrode Position Changes During Respiration with Electrical Impedance Tomography
}

\author{
Jie Zhang ${ }^{1, *}$, Lihong Qin², Tadashi Allen ${ }^{3}$ and Robert P Patterson ${ }^{4}$ \\ ${ }^{1}$ Department of Radiology, University of Kentucky, Lexington, KY 40536 \\ ${ }^{2}$ Department of Therapeutic Radiology, University of Minnesota, Minneapolis, MN 55455 \\ ${ }^{3}$ Department of Radiology, University of Minnesota, Minneapolis, MN 55455 \\ ${ }^{4}$ Department of Physical Medicine and Rehabilitation, University of Minnesota, Minneapolis, MN 55455
}

\begin{abstract}
For pulmonary applications of Electrical Impedance Tomography (EIT) systems, the electrodes are placed around the chest in a 2D ring, and the images are reconstructed based on the assumptions that the object is rigid and the measured resistivity change in EIT images is only caused by the actual resistivity change of tissue. Structural changes are rarely considered. Previous studies have shown that structural changes which result in tissue/organ and electrode position changes tend to introduce artefacts to EIT images of the thorax. Since EIT reconstruction is an ill-posed inverse problem, any small inaccurate assumptions of object may cause large artefacts in reconstructed images. Accurate information on structure/electrode position changes is a need to understand factors contributing to the measured resistivity changes and to improve EIT reconstruction algorithm. Our previous study using MRI technique showed that chest expansion leads to electrode and tissue/organ movements but not significant as proposed. The accuracy of the measurements by MRI may be limited by its relatively low temporal and spatial resolution. In this study, structure/electrode position changes during respiration cycle in patients who underwent chest CT scans are further investigated. For each patient, sixteen fiduciary markers are equally spaced around the surface, the same as the electrode placement for EIT measurements. A CT scanner with respiration-gated ability is used to acquire images of the thorax. CT thoracic images are retrospectively reconstructed corresponding temporally to specific time periods within respiration cycle (from $0 \%$ to $90 \%$, every $10 \%$ ). The average chest expansions are $2 \mathrm{~mm}$ in anterior-posterior and $-1.6 \mathrm{~mm}$ in lateral directions. Inside tissue/organ move down $9.0 \pm 2.5 \mathrm{~mm}$ with inspiration of tidal volume $(0.54 \pm 0.14$ liters $)$, ranging from $6 \mathrm{~mm}$ to $12 \mathrm{~mm}$. During normal quiet respiration, electrode position changes are smaller than expected. No general patterns of electrode position changes are observed. The results in this study provide guidelines for accommodating the motion that may introduce artefacts to EIT images.
\end{abstract}

Keywords: Respiration, Electrical Impedance Tomography, EIT, chest expansion, organ movement, electrode position change, computed tomography, CT.

\section{INTRODUCTION}

Electrical impedance tomography (EIT) has shown promise in monitoring pulmonary ventilation and fluid volume changes [1-2]. As the largest organ in the thorax, the lung resistivity change may be more accurately measured by EIT images compared to other thoracic organs or tissues [3]. The advantages of EIT measurement are that it is non-invasive, relatively inexpensive and non-cumbersome compared with other imaging techniques such as Computed Tomography (CT), Magnetic Resonance Imaging (MRI), etc. However, the poor spatial resolution of EIT significantly limits its applications. Different from other imaging techniques, where

*Address correspondence to this author at the Division of Radiological Medical Physics, Department of Radiology, University of Kentucky, 800 Rose Street, HX311A, Lexington, KY 40536; Tel: 859-323-2146;

Fax: 859-257-4457; Email: jnzh222@uky.edu tissues/organs and the changes of anatomic structures can be perceived, EIT image shows resistivity distribution or resistivity changes relative to a reference frame within a body from which no specific organ/tissues can be directly identified.

For pulmonary application of EIT systems, the electrodes are placed around the chest in a 2D ring, and the images are reconstructed based on the assumption that the object is rigid. It has often been assumed that the measured resistivity change in EIT images is only caused by the actual resistivity change of tissue or in some cases the volume change of the vessel. Structural changes which results in electrode position changes are rarely considered. During inspiration, the chest expands as much as $10 \%$ in the anterior-posterior (AP) direction [4], and the tissues/organs inside the thorax move down with the diaphragm. Since image reconstruction of EIT is an ill-posed inverse problem, EIT is noise sensitive. Any small changes in the structure and/or electrode positions will 
cause errors in measurements therefore introduce artefacts in the image.

Simulation study using thoracic models as virtual phantom has played a major role in understanding factors that contribute to the measured resistivity changes of EIT image. Adler et al (1996) [5] studied the influence of chest expansion during respiration on EIT images using a 2D thorax finite element model. The results showed that chest expansion contributed to the measured change in conductivity and accounted for $2 \%$ to $20 \%$ of the reconstructed image amplitude in a broad zone at the center of the image. Another study using a 3D thorax finite different model showed that both chest expansion and tissue movements during respiration significantly contributed to the lung resistivity changes [6,7]. The average resistivity changes in the lung region caused by chest expansion were between $0.65 \%$ and $18.31 \%$. The artefacts in the center ranged from $-2 \%$ to $31 \%$ of the image magnitude.

The investigation using thoracic models is potentially limited due to lack of in vivo measurements of structural changes as well as corresponding electrode position changes. Any small error in the assumed electrode position changes may result in false estimations of artefacts in EIT image. Therefore, accurate information on structure/electrode position changes is a need to understand factors contributing to the measured resistivity changes and to improve EIT reconstruction algorithms. Our previous study using Magnetic Resonance Imaging (MRI) technique showed that chest expansion during respiration leads to electrode and tissue/organ movements but not significant as mentioned above. This may be partially due to relatively low temporal resolution of
MRI [8]. In this study, we further investigate thoracic structure/electrode position changes during respiration cycle using Computed Tomography (CT) imaging technique. With its superior temporal and spatial resolution, we expect that CT will generate more accurate measurements of electrode and tissue/organ movements during respiration than those using MRI. The accurate measurements of these factors may lead to accurate estimation of errors to EIT image of the lung, which need further study.

\section{MATERIAL AND METHODS}

\subsection{Data Acquisition}

A Philips 16 slice large bore Computed Tomography (CT) scanner (Philips Medical Systems, Philips) located at the VA Minneapolis hospital with respiration-gate was used to acquire thoracic images. The study was approved by IRB of the VA Minneapolis hospital. The images were acquired from patient who underwent CT scans for the planning of radiation therapy. As shown in Fig. (1), sixteen fiducial markers (Radiation Products Design, Inc, Albertville, MN, USA) were equally spaced around the surface of the patient's chest, the same as the electrode placement for EIT measurements [9]. No current was injected and no voltage was measured. The markers were used only for identification of electrode positions.

CT thoracic images were acquired from five male patients. Cross-sectional CT images covering from the neck to the lower abdomen were retrospectively reconstructed corresponding temporally to specific time periods (Fig. (1), from $0 \%$ to $90 \%$, every $10 \%$ ) within respiration cycle. The image
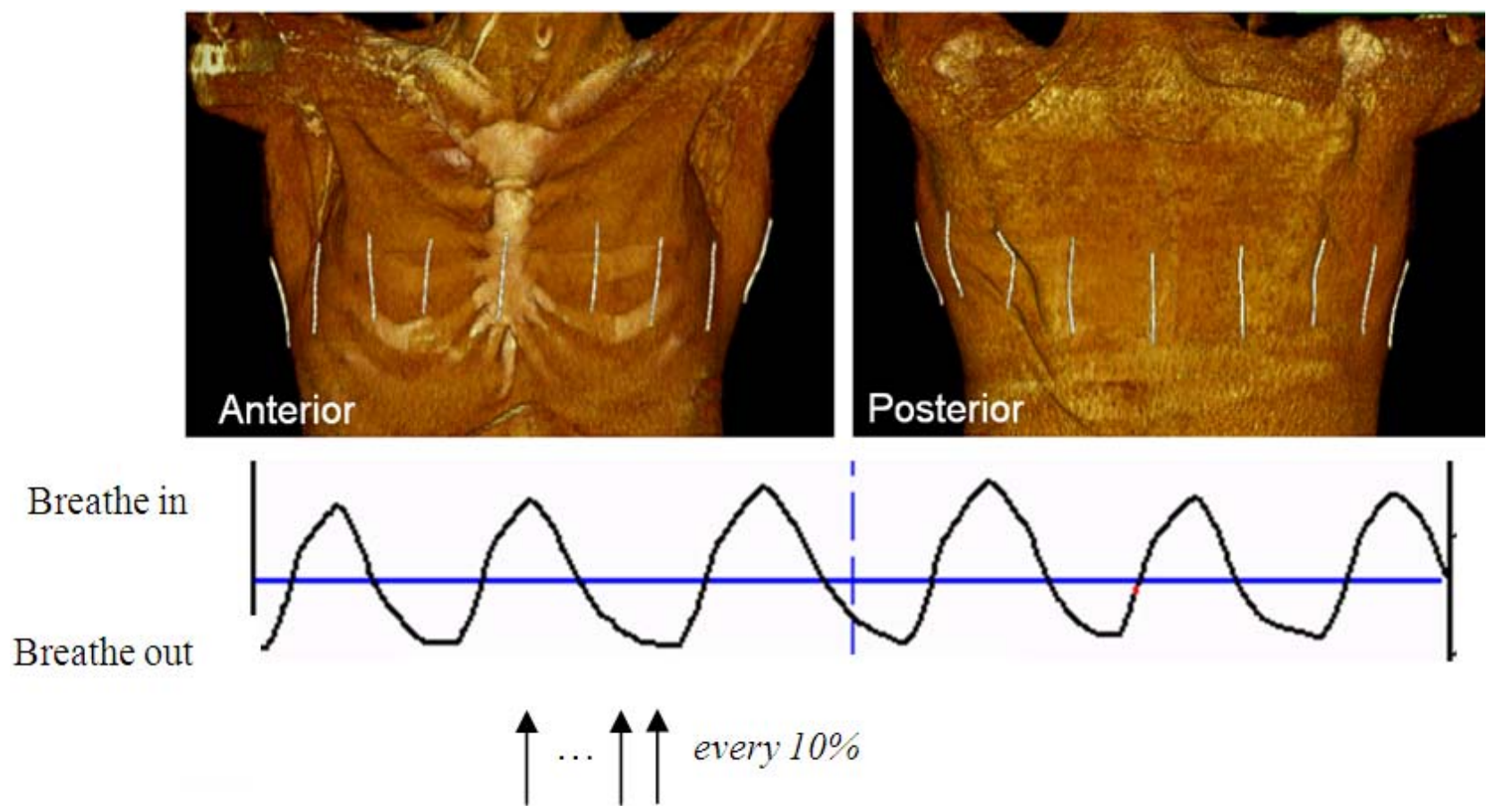

Fig. (1). CT experiment setups and image reconstructed corresponding to respiration cycle. Sixteen fiduciary markers were equally spaced around the surface of the patient's chest, the same as the electrode placement for EIT measurements. No current was injected and no voltage was measured. The markers are used only for identification of electrode positions. Transverse CT images covering from the neck to the lower abdomen were retrospectively reconstructed corresponding temporally to specific time periods, from $0 \%$ to $90 \%$, every $10 \%$ within respiration cycle. 
resolution is approximately $1.0 \mathrm{~mm} \times 1.0 \mathrm{~mm} \times 3 \mathrm{~mm}$. Ten sets of thoracic images for each patient were obtained. These image datasets were analyzed to obtain the chest expansion/electrode position and tissue movements during respiration cycle.

\subsection{Air Volume Measurement}

For each patient, air volume changes during respiration cycle were calculated using mean CT number and total lung volumes, based on [10] equation (1)

$$
\frac{\text { volume }_{\text {gas }}}{\text { volume }_{\text {gas }}+\text { volume }_{\text {tissue }}}=\frac{\text { meanCTnumber }_{\text {observed }}}{\text { CTnumber }_{\text {gas }}-\text { CTnumber }_{\text {water }}}
$$

The lung volumes and mean CT number at each phase during respiration cycle were measured using the Analyze 10 software (AnalyzeDirect, Inc., KS, USA). First, the regions of interest (lungs) were segmented automatically from surrounding regions by setting up threshold levels. Then the segmented lung regions were visually evaluated and manually adjusted to ensure the regions inside the lungs were totally included. Finally the mean CT number and volumes of the lungs were calculated by sampling all the slices that included the lung regions.

During a normal respiration cycle, the tidal volume (TV) refers change in the lung air volume between the end of normal inspiration and expiration while Functional Residual Capacity (FRC) corresponds to lung air at the end of normal expiration.

\subsection{Electrode Position Change Analysis}

The spacing between each adjacent electrode pair was calculated from measurements of $\mathrm{x}, \mathrm{y}$ coordinators of fiducial makers in the transverse CT images. The level where the measurements were made was chosen approximately at the level of nipple line. Since the displacement change between adjacent electrode pair did not correlate well with air volume changes during respiration (see Fig. 3 in the results), the calculated displacement of each adjacent electrode pair for every phase (from $0 \%$ to $90 \%$ ) was performed first. Then the largest and smallest displacements were chosen to calculate the spacing change during the normal respiratory cycle. The average and standard deviation of maximum changes for each electrode pair of five patients were also calculated and presented.

\subsection{Chest Expansion and Tissue/Organ Movement Measurement}

The lungs, liver, pancreas, among other organs within thorax and abdomen, are known to move with breathing. During inhalation, the diaphragm contracts, the lung descends and the abdomen is forced down and forward, and the rib cage is lifted. The intercostals muscles contract to pull and rotate the ribs, resulting in increasing both the lateral and AP diameters of the thorax.

The chest expansion was described using the changes in AP and lateral directions. The chest expansions in AP and lateral directions at TV were measured and compared to those at the phase of FRC.

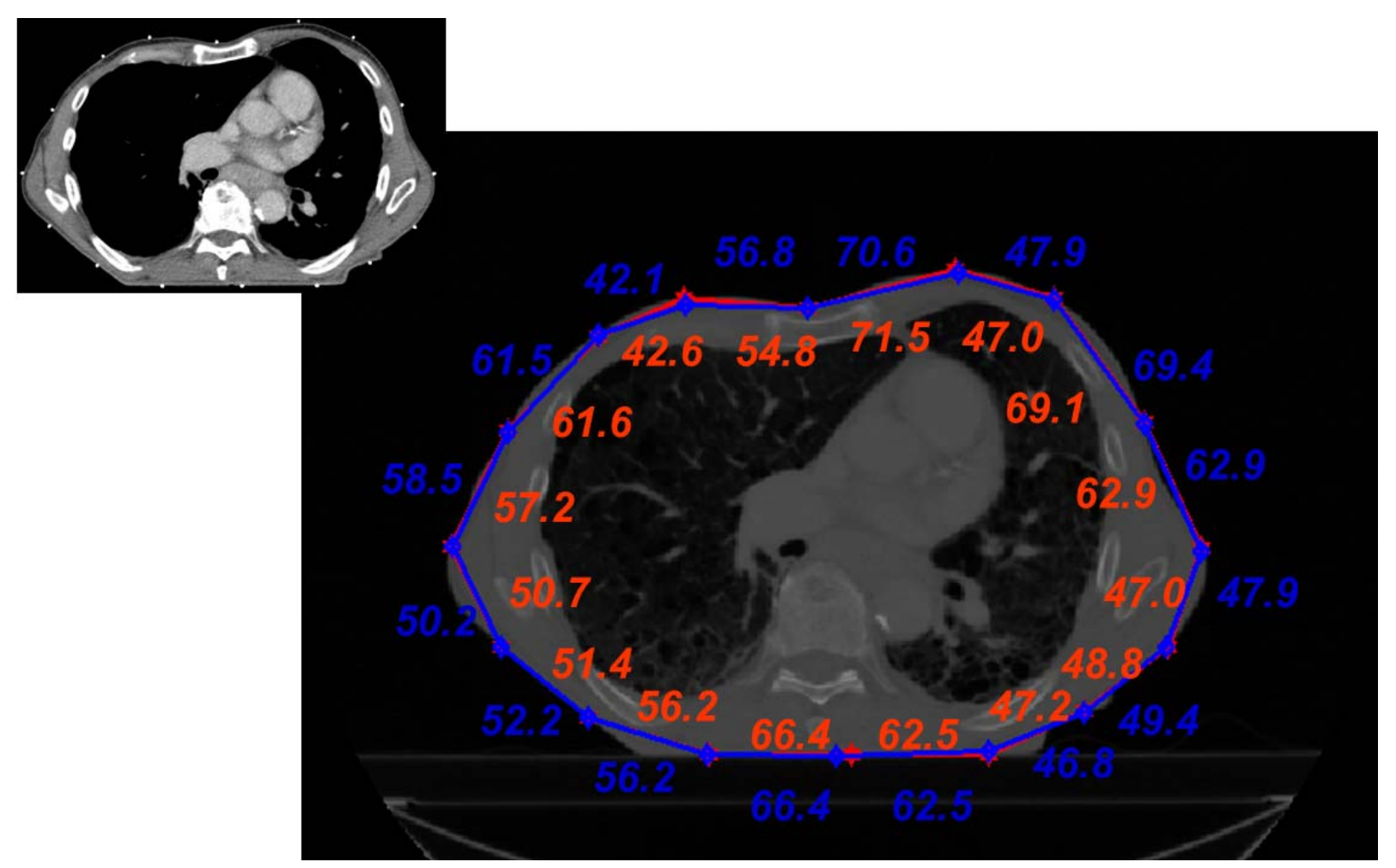

Fig. (2). An example of displacements of adjacent electrode pairs between FRC+TV and FRC. The measurements were made approximately at the nipple line level. Sixteen markers are equally spaced around the thorax, the same as the electrode placement for EIT measurements. The red numbers (inside circle) are at FRC and the yellow numbers (outside circle) are at FRC+TV 
The large tissue/organs movement between FRC+TV and FRC were measured by visually observing tissue/organ in the transverse CT images at different levels of the thorax.

\section{RESULTS}

\subsection{Electrode Position Changes}

Fig. (2) shows an example of displacements of adjacent electrode pairs during normal TV breathing. The measurements were made approximately at the nipple line level. Fig. (3) shows spacing of adjacent electrode pairs varies corresponding to air volume changes during respiration cycle. For supine position, the electrode position changes are mainly observed in the electrodes placed at anterior regions of the chest. Therefore, in Fig. (3), only spacing variations for a few electrode pairs in anterior surface are shown for demonstration purpose. From Fig. (3) we can see that electrode position change doesn't follow air volume changes during respiration cycle.

Fig. (4) shows the maximum displacement changes of each adjacent electrode pairs for each patient. Average displacement changes and the standard deviations of each adjacent electrode pair is also presented beside individual values.

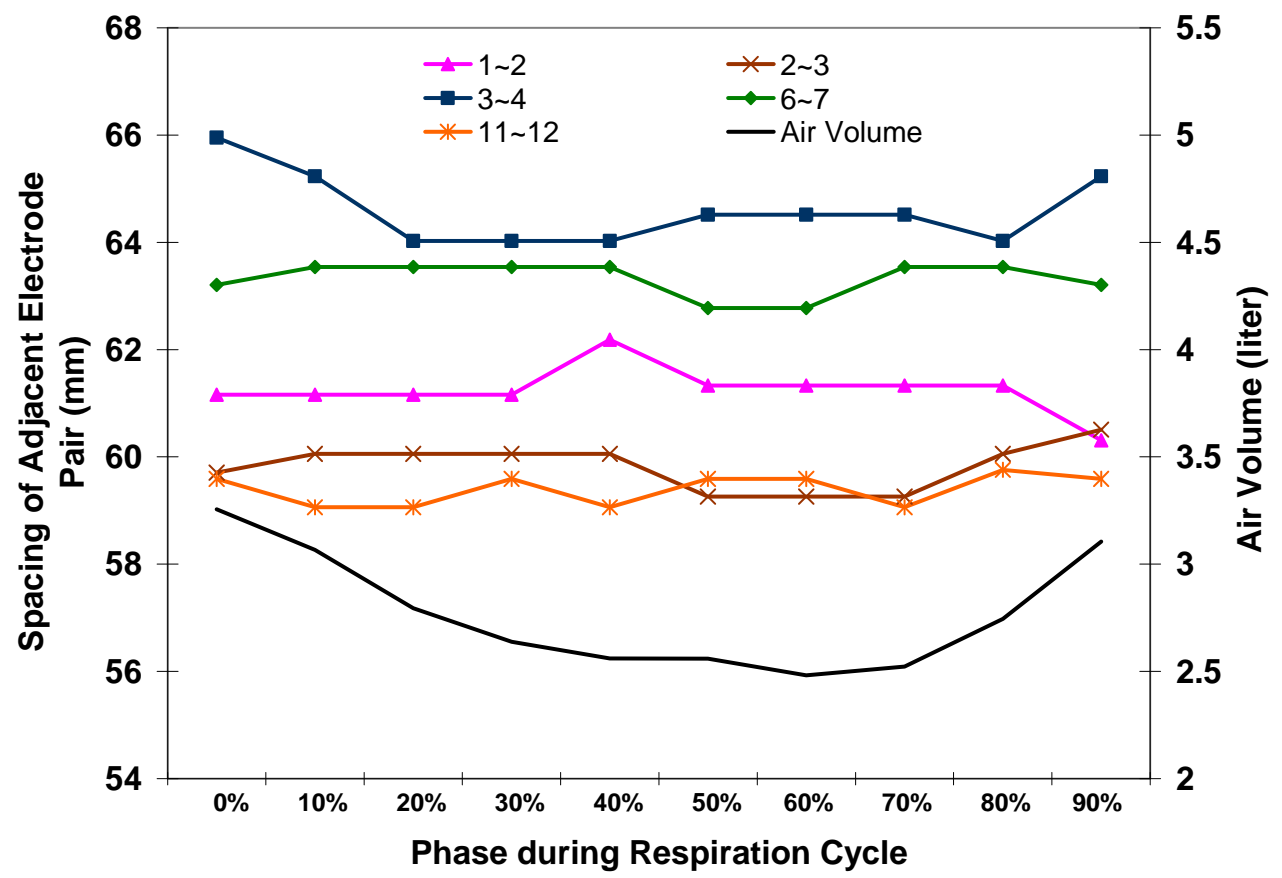

Fig. (3). An example shows that spacing between adjacent electrodes changes corresponding to air volume changes during respiration cycle. Spacing for several electrode pairs in anterior surface are shown for demonstration purpose.

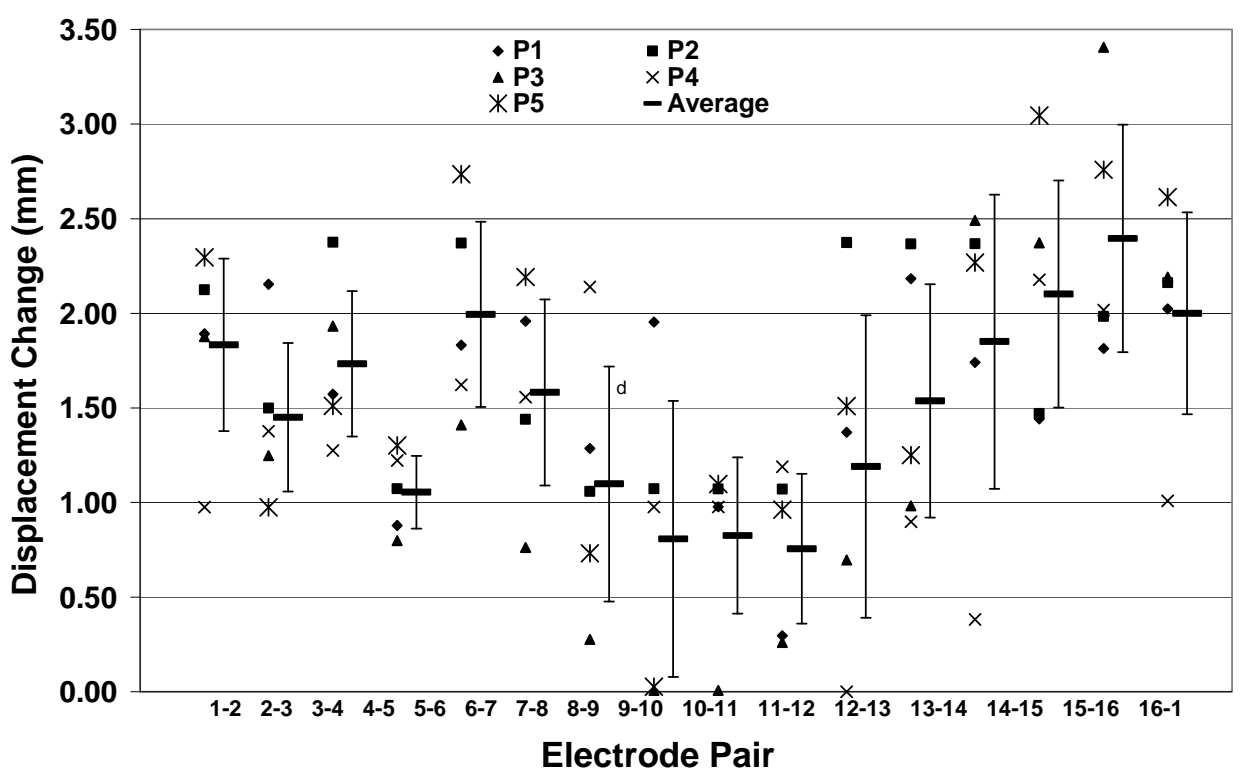

Fig. (4). The maximum spacing changes of each electrode pair for each patient (P1 to P5) (Right dots) and average displacement changes and standard deviations of five patients (left bar). 

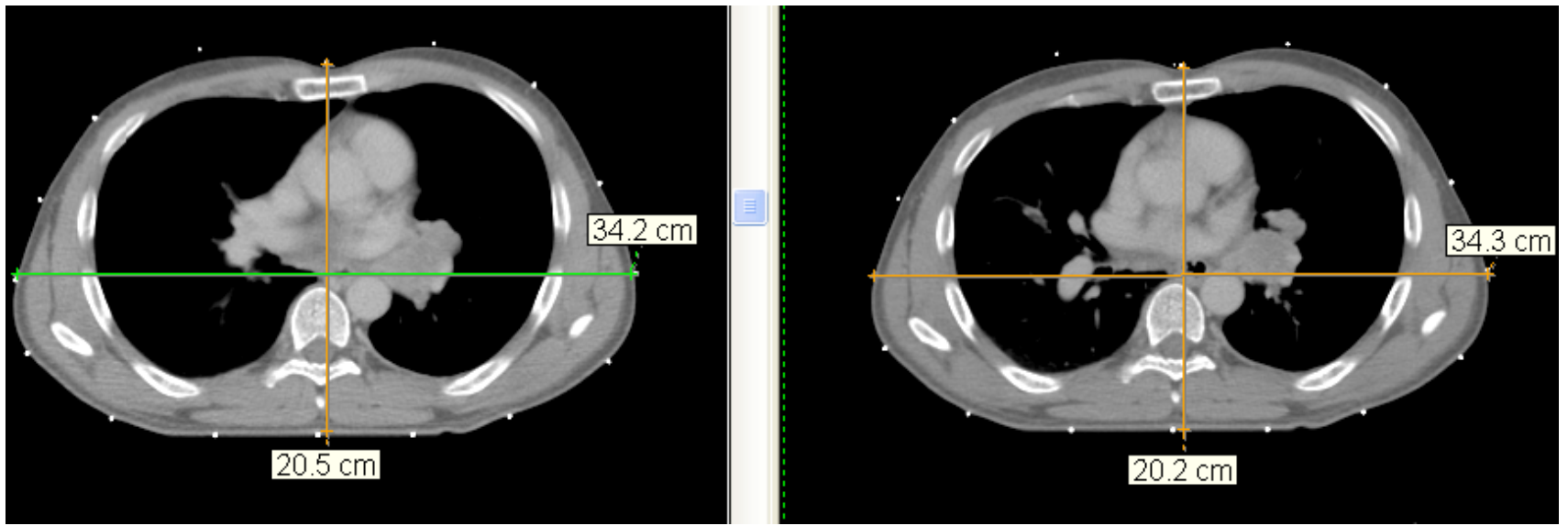

Fig. (5). An example shows that chest expansion in AP and lateral directions are measured. The measurements were made at the same level at FRC+TV (left image) and FRC (right images).

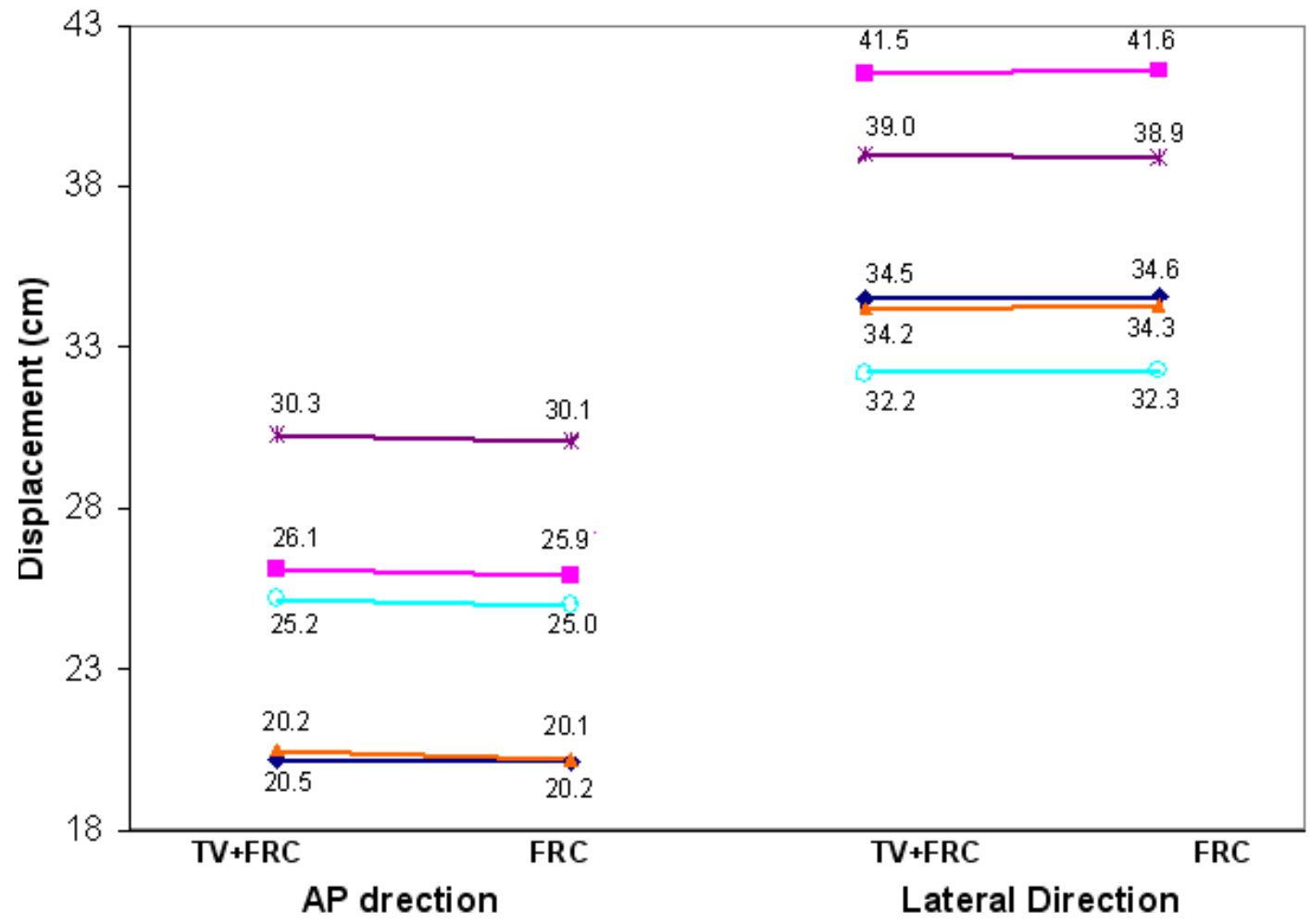

Fig. (6). Chest expansion in anterior-posterior (AP) and lateral directions for 5 patients. The measurements are made according to Fig. (5). The average changes are $2 \mathrm{~mm}$ in AP direction and $-1.6 \mathrm{~mm}$ in lateral direction, corresponding to average air volume change from FRC+TV of 2.81 liters to FRC of 2.27 liters, with a TV of 0.54 liters.

\subsection{Chest Expansion and Tissue/Organ Movements}

Fig. (5) shows an example that the chest expansions in AP and lateral directions are measured. The left image is at FRC+TV while the right image at FRC.

Fig. (6) shows chest expansion in AP and lateral directions for 5 patients. The average changes are $2 \mathrm{~mm}$ in AP direction and $-1.6 \mathrm{~mm}$ in lateral direction, corresponding to average air volume change from 2.81 liters to 2.27 liters, with tidal volume of 0.54 liters.
Fig. (7) shows an example of tissue/organ movements in the AP direction. CT image at FRC+TV (upper left) and that at FRC (upper right) are from the same transverse location but with different air volumes, 3.17 liter vs. 2.65 liter. Obviously inside tissue/organs changed. We visually examined CT images at other transverse locations (lower image) to find an image where inside tissues/organs were close to those in the image at FRC+TV. The location difference was measured as tissue/organ movement with inspiration from FRC to FRC+TV.

Our measurements for five patients show that tissue/organ movements are $9.0 \pm 2.5 \mathrm{~mm}$, ranging from $6 \mathrm{~mm}$ to $12 \mathrm{~mm}$. 

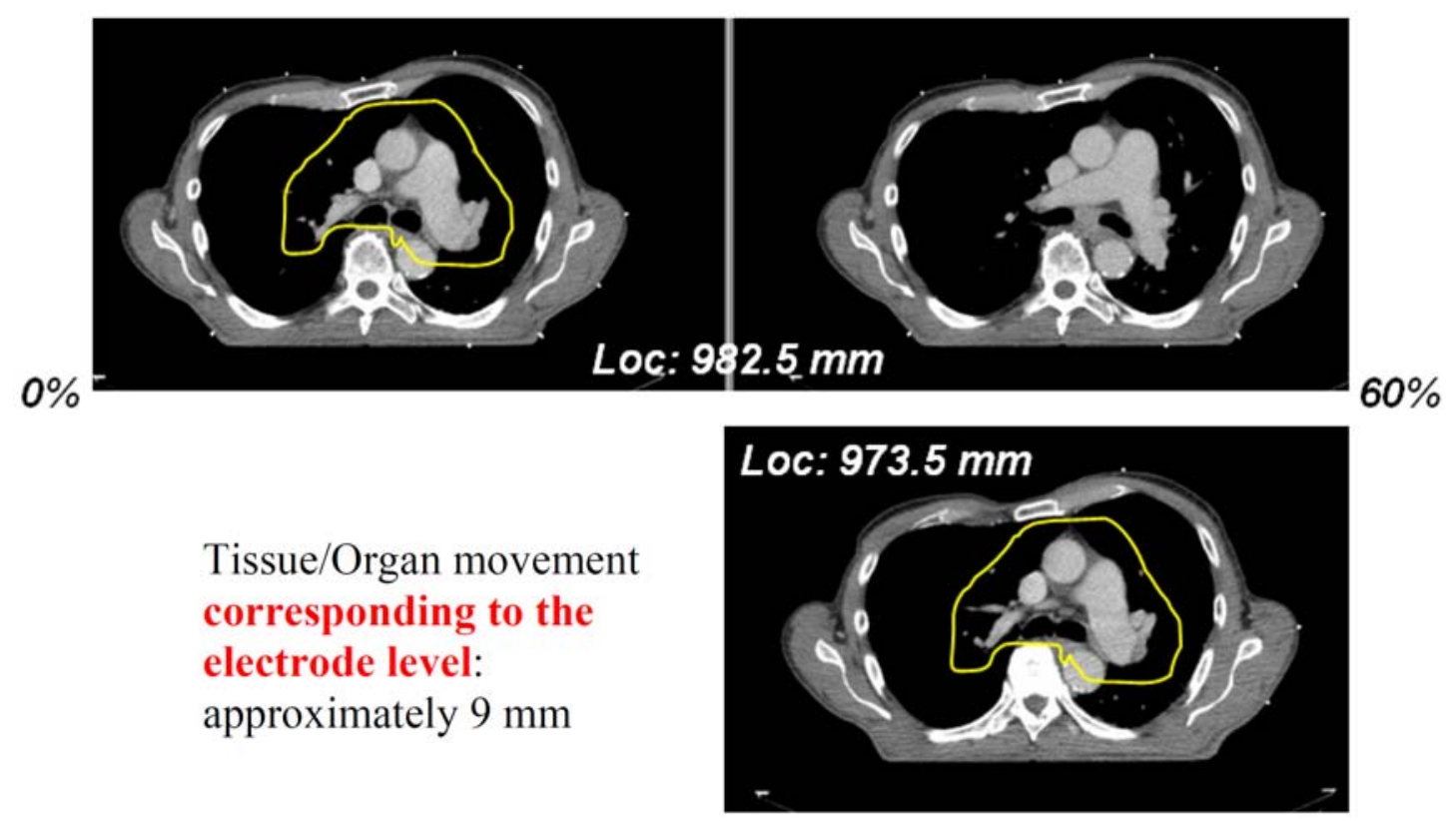

Fig. (7). An example of tissue/organ movements between CT image at FRC+TV (phase of 0\%, upper left) and that at FRC (phase of 60\%, upper right). The images are from the same transverse location but with different air volumes, 3.17 liter (FRC+TV) vs. 2.65 liter (FRC). We visually examined CT images at other transverse locations to find an image where inside tissue/organ (phase of 60\%, lower image) are close to those in the image at FRC+TV. The location difference was measured as tissue/organ movement.

\section{DISCUSSION}

Chest expansion/electrode position changes and tissue/organ movements are obviously one of the potential sources that may cause errors in EIT images of ventilation. The use of in vivo structure and electrode position changes during respiration using human CT thoracic images was more accurate compared to methods used in past studies. The chest expansion and electrode position changes are smaller than the chest expansion used for previous modelling study [5] and those measurements with breath holding at different air volumes [6,7]. The measurement implies the error caused by chest expansion/electrode position changes may not be as significant as estimated by the use of model. An accurate estimation of errors based on in vivo measurements need further study.

CT images can provide more accurate electrode position changes during respiration with its fast acquisition time and the use of retrospective respiration gating technique. Since several respiration cycles are needed to acquire the images of the thorax, variability in the rate of respiration cycle may cause variation in the gating points. For quiet normal respiration, the variability should be small. Another possible cause of error is to find the fiducial maker position for the measurement. The fiducial markers used in this study are nonmetallic. It can provide a clear and accurate reference point with no spray artefacts. In addition, the diameter of fiducial marker was $0.5 \mathrm{~mm}$, which was smaller than CT pixel size of approximately $1 \mathrm{~mm}$. In this way, only one pixel with large CT number shows the electrode position. This helps maintain accuracy of measurements, independent of operators who made measurements. In addition, since the changes between adjacent electrodes during respiration rather than absolute positions were measured, the measurements are also independent of operators who placed electrodes around the chest. Previous study has shown that approximately 7\% accuracy could be approached to measure diameter changes of a $8 \mathrm{~mm}$ tube during cardiac cycle using a CT scanner [11]. Compared to cardiac cycle, respiration cycle is much slower and the distances between two adjacent electrodes (Fig. 2) are much larger than $8 \mathrm{~mm}$. Therefore a higher accuracy $(<7 \%)$ could be expected in this study.

During normal respiration, tissue/organ could move up to $12 \mathrm{~mm}$. Large movement is expected to occur in the lower part of the lungs. With the electrodes placed at 2D rings from where EIT image is reconstructed, inside tissue/organ moving out of the imaging plane during respiration may introduce large artefact to EIT images of the thorax, depending on the electrode levels.

Breathing pattern characterization measurements have been distinguished by posture (upright, prone, supine, lateral decubitus), breathing type (chest or abdominal), and depth of respiration (shallow, normal, deep). In this study, patients were in supine position with normal quiet respiration. The average air volume change during inspiration and expiration was 0.54 liters, which is similar to what is reported in the literature [12]. The marker used for monitoring respiration was put on the patient's chest. However, no breathing types were monitored or controlled. This is more practical for EIT applications. It is worth noting that there are significant differences in organ motion during quiet (shallow) and deep breathing.

One limitation of this study is that we only investigated electrode position changes and tissue/organ movements at supine position, due to the limitation of CT scanner. However, since the most promising applications of EIT system is continuous pulmonary monitoring, especially for monitoring patients being treated with mechanical ventilation, supine 
position may be the most appropriate body position to be considered.

This study leads to the following conclusion: there are no general patterns of electrode position changes, tissue/organ movements that can be assumed for a particular subject prior to observation. Ideally the respiratory compensation procedures and EIT reconstruction algorithms should be adaptable to each patient's particular breathing behavior. The experimental observations on electrode position changes and organ motion due to respiration presented in this study are more detailed and accurate than have been reported in the past. These data can be used in the future when developing and evaluating various reconstruction algorithms.

\section{CONFLICTS OF INTEREST}

The authors confirm that this article content has no conflicts of interest.

\section{ACKNOWLEDGMENTS}

We would like to thank Minnesota Supercomputing Institute at the University of Minnesota for providing the image analysis software.

\section{REFERENCES}

[1] I. Frerichs, "Electrical impedance tomography (EIT) in applications related to lung and ventilation: a review of experimental and clinical activities”, Physiol. Meas., vol. 21, pp. R1-R21, 2000.
[2] I. Frerichs, J. Hinz, P. Herrmann, G. Weisser, G. Hahn, T. Dudykevych, M. Quintel and G. Hellige, "Detection of local lung air content by electrical impedance tomography compared with electron beam CT”, J. Appl. Physiol., vol. 93, pp. 660-666, 2002.

[3] R. P. Patterson and J. Zhang, "Evaluation of an EIT reconstruction algorithm using finite difference human thorax models as phantoms”, Physiol. Meas., vol. 24, pp. 467-75, 2003.

[4] A. Detroyer and S. H. Loring, "Respiratory muscle action Handbook of Physiology", (Bethesda, MD: American Physiological Society), 1985 p. 3.

[5] A. Adler, R. Guardo and Y. Berthiaume, "Impedance imaging of lung ventilation: do we need to account for chest expansion?”, IEEE Trans. Biomed. Eng., vol. 43, pp. 414-20, 1996.

[6] J. Zhang, "Determination of absolute lung resistivity using electrical impedance tomography” PhD Dissertation, University of Minnesota, 2004

[7] J. Zhang and R. P. Patterson, "EIT images of lung ventilation: what contributes to the resistivity changes?”, Physiol. Meas., vol. 26, pp. s81-s92, 2005.

[8] J. Zhang, L. H. Qin, T. Allen and R. P. Patterson, "In Vivo measurements of Structure/ Electrode position changes during respiration for electrical impedance tomography”, J. Phys.: Conf. Ser. 224 012052 doi:10.1088/1742-6596/224/1/012052, 2010

[9] D. C. Barber and A. D. Seagar, "Fast reconstruction of Resistance Images”, Clin. Phys. Physiol. Meas., vol. 8, pp. 47-54, 1987.

[10] L. Gattinoni, P. Caironi, P. Pelosi, and L. R. Goodman, "What has Computed Tomography taught us about the acute respiratory distress syndrome?”, Am. J. Respir. Crit. Care Med., vol. 164, 2001 pp. 1701-1711.

[11] J. Zhang, J. G. Fletcher, T. J. Vrtiska, A. Manduca, J. L. Thompson, M. L. Raghavan, R. J. Wentz and C. H. McCollough, "LargeVessel Distensibility Measurement with Electrocardiographically Gated Multidetector CT: Phantom Study and Initial Experience1”, Radiology, vol. 45, no. 1, pp. 258-266, 2007.

[12] E. P. Widmaier, H. Raff, K. T. Strang, Human Physiology: the mechansms of body function, Mcgraw-Hill Ryerson, Ltd. 2003: pp. 480-483. 